\title{
Molecular genetic analysis and phenotypic characteristics of a consanguineous family with glycogen storage disease type Ia
}

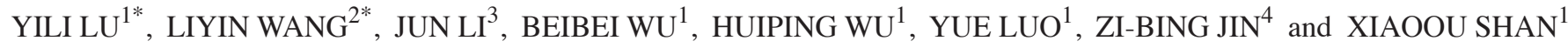 \\ ${ }^{1}$ Department of Pediatrics, The Second Affiliated Hospital of Wenzhou Medical University, Wenzhou, Zhejiang 325027; \\ ${ }^{2}$ College of Basic Medical Sciences, Capital Medical University, Beijing 100069; ${ }^{3}$ Department of Hand Surgery, \\ Wenzhou Donghua Hospital; ${ }^{2}$ Division of Ophthalmic Genetics, Laboratory for Stem Cell and Retinal Regeneration, \\ The Eye Hospital of Wenzhou Medical University, Wenzhou, Zhejiang 325027, P.R. China
}

Received September 17, 2015; Accepted June 29, 2016

DOI: $10.3892 / \mathrm{mmr} .2016 .5617$

\begin{abstract}
Glycogen storage disease type-Ia (GSD-Ia) is a rare autosomal recessive disease caused by a mutation in the gene encoding glucose-6-phosphate- $\alpha$ (G6PC). The present study reported the case of a 3 -month-old female Chinese patient with GSD-Ia born to consanguineous parents. The aim of the present study was to identify the precise mutation of the G6PC gene associated with this family and to describe the phenotypic characteristics of the patient. A comprehensive examination was performed on the patient, including physical examination, vein blood gas analysis, abdominal sonography and biochemical analyses. In addition, gene sequencing was performed on the coding region of the G6PC gene to identify the mutation. The patient was diagnosed with GSD-Ia and a G6PC missense mutation of c.518T >C (p.L173P) located in a highly conserved area was identified. The mutation is in a non-helical region of the protein, which previous studies have suggested should result in a lesser effect on G6PC enzymatic activity and milder phenotypic characteristics compared with mutations located in helical regions. However, the severity of the disease phenotype in the subject of the present study was inconsistent with that predicted from her genotype. The patient suffered from serious hypoglycemia, lactic acidosis, increased
\end{abstract}

Correspondence to: Dr Xiaoou Shan, Department of Pediatrics, The Second Affiliated Hospital of Wenzhou Medical University, 109 West Xueyuan Road, Wenzhou, Zhejiang 325027, P.R. China E-mail: sssxooo@sina.com

Dr Zi-Bing Jin, Division of Ophthalmic Genetics, Laboratory for Stem Cell and Retinal Regeneration, The Eye Hospital of Wenzhou Medical University, 270 West Xueyuan Road, Wenzhou, Zhejiang 325027, P.R. China

E-mail: jinzb@mail.eye.ac.cn

*Contributed equally

Key words: glycogen storage disease, glucose-6-phosphatase, phenotype-genotype correlation, glucose-6-phosphate- $\alpha$ gene, mutation triglycerides, hepatic dysfunction, clear hepatomegaly and nephromegaly. The incidence of the p.L173P mutation may be relatively high in the Chinese population. Knowledge of the various phenotypic presentations of the p.L173P mutation may beneficial for future investigations.

\section{Introduction}

Glycogen storage disease-type I (GSD-I), first described by von Gierke in 1929 (1), is an inherited deficiency of the glucose-6-phosphatase (G6Pase) complex. G6Pase is composed primarily of glucose-6-phosphatase- $\alpha$ (G6PC) and glucose-6-phosphate translocase (G6PT) (2). Abnormalities in G6PC and G6PT result in GSD-Ia and GSD-Ib, respectively, which are the primary subtypes of GSD-I (2).

G6PC is a glycoprotein anchored in the endoplasmic reticulum membrane by nine transmembrane helices. G6PC is vital for the maintenance of glucose homeostasis as it catalyzes the final step in the glycogenolytic and glyconeogenic pathways $(2,3)$, and is therefore present in numerous glucose-generating organs, including the liver, kidney and small intestine $(4,5)$. G6PC deficiency or abnormality may result in GSD-Ia $(2,6)$. GSD-Ia is characterized by hypoglycemia, growth retardation, lactic acidosis, high levels of lipids, hepatomegaly and nephromegaly. Due to these clinical manifestations, patients with GSD-Ia are typically diagnosed in childhood, and the diagnosis may be confirmed using gene sequencing to confirm the presence of mutations in $G 6 P C(6,7)$. G6PT, the protein involved in GSD-1b, is a transporter that functions to facilitate glycogen entry into the cell. Similar to GSD-Ia, GSD-Ib is characterized by hypoglycemia, high levels of lipids and hepatomegaly. However, patients with GSD-Ib may additionally suffer from impaired neutrophil function and neutropenia, resulting in recurrent bacterial infections (2).

GSD-Ia is responsible for $>80 \%$ of GSD-I cases (4). The overall incidence of GSD-Ia in the population is reported to be 1 in 100,000, with a carrier frequency of $\sim 1$ in 150 (2). To date, 86 disease-causing mutations in G6PC have been identified (6), including missense mutations, nonsense mutations, frameshifts resulting from insertions and deletions, and rare gene rearrangements (6), with missense mutations being the 
most common (6). Although GSD-Ia is a pan-ethnic disease, the incidence of specific mutations varies between ethnic populations (4). For example, the c.648G $>\mathrm{T}$ mutation is prevalent in East Asians (4), whereas c. $247 \mathrm{C}>\mathrm{T}$ is common in the Jewish population (6). G6PC mutations may be classified into three categories based on their predicted topological position: Non-helical, helical and active site. Typically, non-helical mutations have a lesser effect on enzymatic activity compared with helical mutations (8), and in vitro experiments have demonstrated that G6PC containing non-helical mutations may retain enzymatic activity (9). However, the precise association between mutations and disease severity remains to be fully elucidated. In the present study, a missense mutation of G6PC was identified in a 3-month-old female patient from a consanguineous family. The association between the G6PC genotype and disease phenotype in this patient is in contrast to that previously described (8). The results of the present study suggested that the prevailing view that mutations in non-helical regions of $G 6 P C$ result only in mild phenotypic manifestations may require revision.

\section{Materials and methods}

Patient characteristics. The 3-month-old female patient with hypoglycemia, hepatocelluar dysfunction and lactic acidosis was groaning frequently. The patient was in a clear state of mind and not convulsing. The parents brought her to The Second Affiliated Hospital of Wenzhou Medical University (Wenzhou, China), for an emergency visit, following which she was admitted to the Department of Pediatrics. She weighed $6 \mathrm{~kg}$ and was $60 \mathrm{~cm}$. The patient did not suffer from severe recurrent infections. Her parents are first cousins (Fig. 1A). There was no history of note. A comprehensive examination of the patient was performed, including a physical examination, vein blood gas analysis, an electrolyte test, an abdominal sonography and biochemical analyses.

Molecular screening. Informed consent was obtained from everyone involved in the present study. The study protocol was approved by The Second Affiliated Hospital of Wenzhou Medical University Ethics Committee (Wenzhou, China). Genomic DNA was isolated from peripheral blood samples using a genomic DNA extraction kit (RelaxGene Blood DNA system; Tiangen Biotech Co., Ltd., Beijing, China), according to the manufacturer's protocols. The five exons and flanking introns of the $G 6 P C$ gene were amplified by polymerase chain reaction (PCR) with forward, AGTCCACTTCGA ACAGCCAG and reverse, CCGATCGCGTCATGGAAA AC primers. The $50 \mu 1$ total reaction volume contained $0.4 \mu \mathrm{mol} / 1$ of each primer, $0.2 \mathrm{mmol} / 1 \mathrm{dNTPs}, 1 \mathrm{X}$ PCR buffer, 50 ng genomic DNA, and 1 unit Taq DNA polymerase (Takara Biotechnology Co., Ltd., Dalian, China). The PCR cycling conditions consisted of denaturation at $95^{\circ} \mathrm{C}$ for $4 \mathrm{~min}$ followed by 40 thermal cycles of $95^{\circ} \mathrm{C}$ for $30 \mathrm{sec}, 58^{\circ} \mathrm{C}$ for $30 \mathrm{sec}$, and $72^{\circ} \mathrm{C}$ for $42 \mathrm{sec}$. Resulting PCR products were electrophoresed on a $1.5 \%$ agarose gel and purified using a PCR purification kit (AxyPrep PCR Cleanup kit; Hangzhou Axygen Biotechnology Ltd., Hangzhou, China). The PCR products were sequenced using an automated sequencer (ABI Prism 3100 Genetic Analyzer; Applied Biosystems; Thermo
Fisher Scientific, Inc., Waltham, USA), and compared with a reference sequence. The gene sequences surrounding the mutation site in various species were obtained from the National Center for Biotechnology Information (www.ncbi. nlm.nih.gov/pubmed/) and analyzed to determine whether the mutation site was conserved.

\section{Results}

Clinical examination. The 3-month-old female patient was born in a consanguineous family (Fig. 1A). She was groaning frequently. There was no history of note. Physical examination revealed shortness of breath, symmetrical rough breath sounds, regular respiratory rhythm, and abdominal distension with enlarged liver ( $7 \mathrm{~cm}$ below the right costal margin). The spleen was not palpable. An increased level of lactic acid revealed that the patient had lactic acidosis. Vein blood gas analysis revealed that $\mathrm{pH}, \mathrm{PCO}_{2}$ and $\mathrm{HCO}_{3}^{-}$levels were below normal, and that the absolute value of base excess was increased, suggesting metabolic acidosis (Table I). In addition, the shortness of breath exhibited by the patient indicated metabolic acidosis. A fasting blood glucose level of $2.73 \mathrm{mmol} / \mathrm{l}$ indicated that the infant suffered from severe hypoglycemia. Furthermore, increased levels of alanine aminotransferase, aspartate aminotransferase, and $\gamma$-glutamyl transpeptidase suggested liver dysfunction. Additionally, the levels of uric acid and triglycerides were also increased (Table II). Abdominal sonography identified hepatomegaly with enhanced echo texture (Fig. 1B) and nephromegaly (Fig. 1C), with the size of left kidney measured at 72x40 mm and the size of right kidney measured at $71 \times 30 \mathrm{~mm}$. The enlarged organs indicated the severity of the disease. A viral hepatitis serology screen revealed that the patient was positive for hepatitis B surface antigen (461.5), negative for hepatitis B core antibody, and that total immunoglobulin was normal. The patient was negative for human immunodeficiency virus. In summary, the patient's clinical manifestations and test results suggested GSD-Ia.

Diagnosis of GSD-Ia. Gene sequencing revealed that the patient was homozygous for a missense mutation in the G6PC gene, c.518T >C (p.L173P; Fig. 2A), which changed the codon for leucine at position 518 to a codon for proline. Pedigree analysis revealed that the patient's father and mother are heterozygous for this mutation (Fig. 1A). By comparing the $G 6 P C$ sequence across various species, it was determined that the mutation is located in a highly conserved region of the G6PC gene (Fig. 2B). Furthermore, the p.L173P mutation is absent from the $1000 \mathrm{G}$ database (http://www.1000genomes. org/), suggesting it is a very rare mutation. This finding, together with the severe symptoms of the patient, suggested that there is a high probability that the p.L173P mutation results in abnormal G6PC enzyme function. However, this mutation affects a non-helical region of the protein, which previous studies suggest are of less importance compared with the helical regions for enzyme function (8). Therefore, the phenotypic characteristics associated with a mutation in the non-helical region of the protein are expected to be mild. However, in the present study the clinical manifestations were severe. 


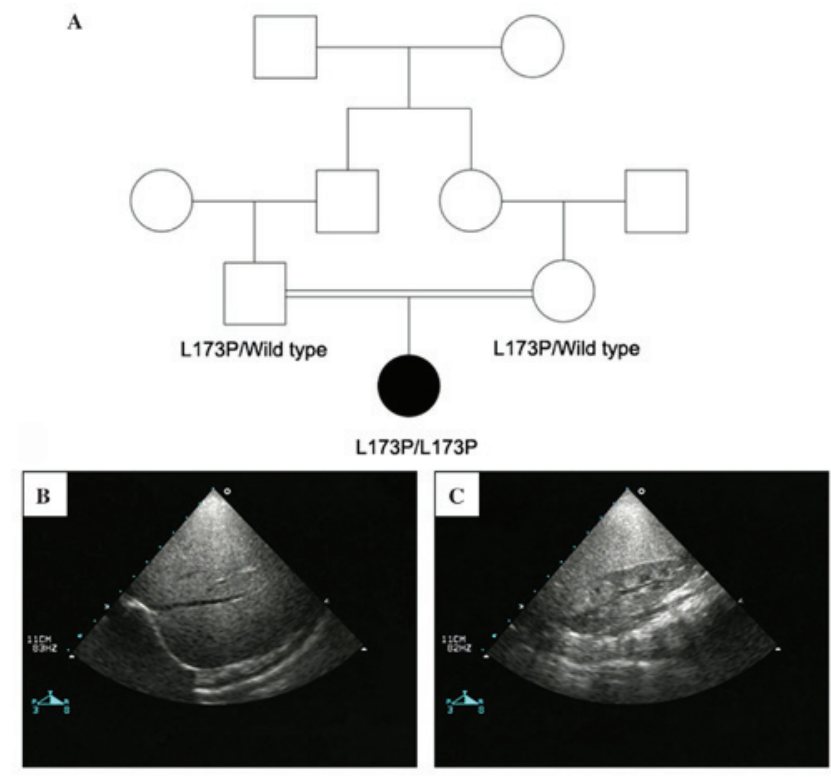

Figure 1. (A) Pedigree analysis. The patient is demarcated in black. The patient was homozygous for the mutation and her parents were heterozygous carriers. (B) Liver sonography revealed enlargement of the liver. (C) Kidney sonography revealed enlargement of the kidneys.

A

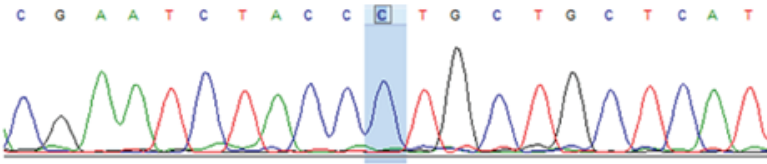

G6PC gene mutation: c.518T $>$ C, p.L173P

\begin{tabular}{l|c|c|c|c|c|c|c|c|c|c|c|} 
Human & L & S & R & I & Y & L & A & A & H & F & P \\
\hline P troglodytes & L & S & R & I & Y & L & A & A & - & F & K \\
\hline M mulatta & L & S & R & I & Y & L & A & A & H & F & P \\
\hline F catus & L & S & R & I & Y & L & A & A & H & F & P \\
\hline M musculus & L & S & R & I & Y & L & A & A & H & F & P \\
\hline D melanogaster & L & S & R & L & Y & L & A & T & E & F & L \\
\hline X tropicalis & L & S & R & I & F & L & A & A & H & F & P
\end{tabular}

Figure 2. Sequence analysis of the $G 6 P C$ gene. (A) Gene sequencing identified a homozygous missense mutation in the $G 6 P C$ gene of the patient: c.518T $>C$, p.L173P. (B) Amino acid alignment of the region of G6PC surrounding the patient's mutation site with the analogous region in various species. G6PC, glucose-6-phosphatase- $\alpha$; L, leucine; P, proline.

\section{Discussion}

G6PC is a 357-amino acid glycoprotein anchored to the ER membrane by nine transmembrane helices, with an enzymatic active site facing the ER lumen (8). The amino-terminus of G6PC is located in the ER lumen, and the carboxyl-terminus is in the cytoplasm $(4,8,10)$. The G6PC gene, located on chromosome $17 q 21$, is a single copy gene composed of five exons (10). The only known cause of GSD-Ia is mutation of G6PC. Mutations have been divided into three categories, non-helical, helical and active site, based on their predicted topological position. In general, non-helical mutations are
Table I. Results of patient vein blood gas analysis.

\begin{tabular}{lccc}
\hline Parameter & Test value & Change & Reference range \\
\hline $\mathrm{pH}$ & 7.26 & $\downarrow$ & $7.35-7.45$ \\
$\mathrm{PCO}_{2}, \mathrm{mmHg}$ & 24.0 & $\downarrow$ & $35-45$ \\
$\mathrm{HCO}_{3}, \mathrm{mmol} / \mathrm{l}$ & 10.3 & $\downarrow$ & $22-27$ \\
$\mathrm{BE}, \mathrm{mmol} / \mathrm{l}$ & -15.1 & $\uparrow$ & $-2-3$
\end{tabular}

BE, base excess.

Table II. Results of patient blood biochemical analysis.

\begin{tabular}{lccc}
\hline Parameter & Test value & Change & Reference range \\
\hline FBG, mmol/l & 2.73 & $\downarrow$ & $3.90-6.10$ \\
Lactic acid, mmol/1 & 12.20 & $\uparrow$ & $0.6-2.20$ \\
ALT, U/l & 102 & $\uparrow$ & $7-40$ \\
AST, U/l & 169 & $\uparrow$ & $13-35$ \\
GGT, U/l & 820 & $\uparrow$ & $7-38$ \\
UA, $\mu$ mol/l & 1035 & $\uparrow$ & $89-357$ \\
TG, mmol/l & 7.90 & $\uparrow$ & $0.56-1.70$ \\
\hline
\end{tabular}

FBG, fasting blood glucose; ALT, alanine aminotransferase; AST, aspartate aminotransferase; GGT, $\gamma$-glutamyl transpeptadase; UA, uric acid; TG, triglyceride.

thought to have a smaller effect on enzyme activity compared with those in transmembrane helices (8). In the present study, the case of a 3-month-old infant with a c.518T >C (p.L173P) non-helical G6PC mutation is reported. According to the prevailing view, the infant would be expected to have mild phenotypic characteristics. However, the clinical manifestations were clear, as the patient suffered from severe hypoglycemia and hepatomegaly. Abdominal sonography revealed nephromegaly. Li et al (11) reported a 3-year-old girl who was not diagnosed with GSD-Ia until the age of 2. The phenotypic characteristics of this patient were fasting hypoglycemia, hepatomegaly, hypercholesterolemia and recurrent epistaxis. The patient did not suffer from nephromegaly or metabolic acidosis. Her biochemical results were improved through a diet of regular uncooked cornstarch and frequent carbohydrate feeds. That patient was not born of a consanguineous union. DNA sequencing identified that the mother had a heterozygous R83H (G327A) mutation and the father had a heterozygous L173P (T597C) mutation (11). Thus, the patient had a p.L173P mutation, as in the present study. While the two girls suffered from identical mutations, the severity of their phenotypic characteristics was very different. The results of the present study suggested that non-helical mutations may result in severe clinical manifestations, and that phenotypic heterogeneity exists in p.L173P G6PC mutations.

GSD-Ia is a pan-ethnic disorder; however, the incidence of mutations varies between ethnicities. To date, no reports of a p.L173P mutation have been presented in any ethnicity other than Chinese. This may indicate that the incidence of the p.L173P mutation is relatively high in the Chinese population. 
In the present study, hypoglycemia and lactic acidosis were under control following administration of intravenous glucose to the infant at a dose of $6 \mathrm{mg} / \mathrm{kg} / \mathrm{min}$. Nocturnal nasogastric infusion of glucose and intermittent oral administration of uncooked cornstarch are the primary dietary therapies for GSD-Ia (12). However, even with dietary therapy, long-term clinical problems may occur. These include growth retardation, hepatomegaly, intermittent hypoglycemia, lactic acidemia, hyperlipidemia, adenoma, gout associated with hyperuricemia, proteinuria and nephrolithiasis with progressive renal failure. Replacement therapy is a promising alternative strategy for GSD-Ia treatment, and may involve administration of G6PC, liver-kidney transplantation or gene therapy. As G6PC is hydrophobic, it is difficult to isolate in an active, soluble form that would facilitate its administration to patients (5). Case reports have revealed that liver-kidney transplantation may rectify the metabolic disorder associated with GSD-Ia $(13,14)$. However, liver-kidney transplantation brings with it associated risks and challenges. Gene therapy, while not yet developed for the clinic, may in the future provide a strategy for the treatment of GSD-Ia $(15,16)$. Previous studies have demonstrated the efficacy of gene therapy in treating mouse models of GSD-Ia $(15,17,18)$. In addition, alternative therapies, including bone marrow-derived myelomonocyte transplantation, may have the potential to restore normal liver function (19).

In conclusion, the present study reports the case of a 3-month-old female Chinese patient with GSD-Ia and identified a G6PC missense mutation of c.518T >C (p.L173P) located in a highly conserved area. The severity of the phenotype was inconsistent with that predicted by her genotype. The incidence of the p.L173P mutation may be relatively high in the Chinese population. Knowledge of the various phenotypic presentations of the p.L173P mutation may be beneficial for future investigations.

\section{Acknowledgements}

The authors appreciate the participation of the patient and her family in the present study. The present study was supported by the Zhejiang Provincial Natural Science Foundation of China (no. Y2100530, awarded to X.S.), the Wenzhou Science and Technology Foundation (no. Y20140358, awarded to X-F.H.) and the Key Scientific and Technological Innovation Team of Wenzhou (no. C20150004, awarded to Z-B.J.).

\section{References}

1. Moses SW: Historical highlights and unsolved problems in glycogen storage disease type 1. Eur J Pediatr 161 (Suppl 1): S2-S9, 2002.

2. Koeberl DD, Kishnani PS, Bali D and Chen YT: Emerging therapies for glycogen storage disease type I. Trends Endocrinol Metab 20: 252-258, 2009.
3. Hutton JC and O'Brien RM: Glucose-6-phosphatase catalytic subunit gene family. J Biol Chem 284: 29241-29245, 2009.

4. Gu LL, Li XH, Han Y, Zhang DH, Gong QM and Zhang XX A novel homozygous no-stop mutation in G6PC gene from a Chinese patient with glycogen storage disease type Ia. Gene 536: 362-365, 2014

5. Resaz R, Vanni C, Segalerba D, Sementa AR, Mastracci L, Grillo F, Murgia D, Bosco MC, Chou JY, Barbieri O, et al: Development of hepatocellular adenomas and carcinomas in mice with liver-specific G6Pase- $\alpha$ deficiency. Dis Model Mech 7: 1083-1091, 2014.

6. Kishnani PS, Austin SL, Abdenur JE, Arn P, Bali DS, Boney A, Chung WK, Dagli AI, Dale D, Koeberl D, et al: Diagnosis and management of glycogen storage disease type I: A practice guideline of the American college of medical genetics and genomics. Genet Med 16: e1, 2014.

7. Wang J, Cui H, Lee NC, Hwu WL, Chien YH, Craigen WJ, Wong LJ and Zhang VW: Clinical application of massively parallel sequencing in the molecular diagnosis of glycogen storage diseases of genetically heterogeneous origin. Genet Med 15: 106-114, 2013.

8. Chou JY and Mansfield BC: Mutations in the glucose-6-phosphatase-alpha (G6PC) gene that cause type Ia glycogen storage disease. Hum Mutat 29: 921-930, 2008.

9. Shieh JJ, Terzioglu M, Hiraiwa H, Marsh J, Pan CJ, Chen LY and Chou JY: The molecular basis of glycogen storage disease type 1a: Structure and function analysis of mutations in glucose-6-phosphatase. J Biol Chem 277: 5047-5053, 2002.

10. Chou JY, Jun HS and Mansfield BC: Glycogen storage disease type I and G6Pase- $\beta$ deficiency: Etiology and therapy. Nat Rev Endocrinol 6: 676-688, 2010

11. Li DZ, Liao C and Tang XW: Prenatal diagnosis of glycogen storage disease type Ia, presenting a new mutation in the glucose-6-phosphatase gene. Prenat Diagn 27: 685-686, 2007.

12. Shah KK and O'Dell SD: Effect of dietary interventions in the maintenance of normoglycaemia in glycogen storage disease type 1a: A systematic review and meta-analysis. J Hum Nutr Diet 26: 329-339, 2013.

13. Belingheri M, Ghio L, Sala A, Menni F, Trespidi L, Ferraresso M, Berardinelli L, Rossi G, Edefonti A and Parini R: Combined liver-kidney transplantation in glycogen storage disease Ia: A case beyond the guidelines. Liver Transpl 13: 762-764, 2007.

14. Marega A, Fregonese C, Tulissi P, Vallone C, Gropuzzo M, Toniutto PL, Baccarani U, Bresadola F, Toso F and Montanaro D: Preemptive liver-kidney transplantation in von Gierke disease: A case report. Transplant Proc 43: 1196-1197, 2011.

15. Yiu WH, Lee YM, Peng WT, Pan CJ, Mead PA, Mansfield BC and Chou JY: Complete normalization of hepatic G6PC deficiency in murine glycogen storage disease type Ia using gene therapy. Mol Ther 18: 1076-1084, 2010.

16. Lee YM, Jun HS, Pan CJ, Lin SR, Wilson LH, Mansfield BC and Chou JY: Prevention of hepatocellular adenoma and correction of metabolic abnormalities in murine glycogen storage disease type Ia by gene therapy. Hepatology 56: 1719-1729, 2012.

17. Lee YM, Pan CJ, Koeberl DD, Mansfield BC and Chou JY: The upstream enhancer elements of the G6PC promoter are critical for optimal G6PC expression in murine glycogen storage disease type Ia. Mol Genet Metab 110: 275-280, 2013.

18. Clar J, Mutel E, Gri B, Creneguy A, Stefanutti A, Gaillard S, Ferry N, Beuf O, Mithieux G, Nguyen TH and Rajas F: Hepatic lentiviral gene transfer prevents the long-term onset of hepatic tumours of glycogen storage disease type 1a in mice. Hum Mol Genet 24: 2287-2296, 2015.

19. Resaz R, Emionite L, Vanni C, Astigiano S, Puppo M, Lavieri R, Segalerba D, Pezzolo A, Bosco MC, Oberto A, et al: Treatment of newborn G6pc(-/-) mice with bone marrow-derived myelomonocytes induces liver repair. J Hepatol 55: 1263-1271, 2011. 